\title{
Mixed Convection Peristaltic Flow of a Eyring-Powell Nanofluid with Magnetic Field in a Non-Uniform Channel
}

\author{
Asha, S. K. ${ }^{1}$, Sunitha, G. ${ }^{1}$ \\ ${ }^{1}$ Department of Mathematics, Karnatak Uni versity, Dharwad-580003, India
}

How to cite this paper: Asha, S. K. \& Sunitha, G. (2018) Mixed Convection Peristaltic Flow of a Eyring-Powell Nanofluid with Magnetic Field in a Non-Uniform Channel. Journal of Applied Mathematics and Computation, 2(8), 332-344. http://dx.doi.org/10.26855/ja mc. 2018.08.003 *Corresponding author: Asha, S. K., Department of Mathematics, Karnatak University, Dharwad-580003, India Email: as.kotnur2008@g mail.com

\begin{abstract}
The present paper deals with the study of mixed convection peristaltic flow of Eyring-Powell Nanofluid with the effect of magnetic field in a non-uniform two dimensional channel. A mathematical modelling is carried out by utilizing long wavelength and low Reynolds number assumptions. Thus peristalsis of Eyring-Powell nanofluid followed through the conservation principles of mass, momentum, energy and concentration has been modelled and Momentum equations involve the combined effects of thermal Grashof number and local nanoparticle Grashof number. The resulting nonlinear system of partial differential equations is solved analytically by employing Homotopy Analysis Method (HAM) and the expressions for velocity, temperature and concentration are determined. The behaviours of Brownian motion parameter $(\mathrm{Nb})$, thermophoresis parameter $(N t)$ on the non- uniform channel are shown graphically and The effect of various physical parameters on the flow characteristics are shown and discussed with the help of graphs.
\end{abstract}

Keywords

Mixed convection, Peristaltic transport, magnetic field, Eyring-Powell nanofluid.

\section{Introduction.}

Peristalsis is a mechanism of fluid transport when a progressive wave of area contraction or expansion propagates along its length. The peristalsis is derived from Latin word and comes from the Greek peristallein. The mechanism of peristalsis is used for pumping physiological, industrial and other forms of fluids from one place to another. This phenomenon widely occurs in several physiological, industrial and biomedical applications such as swallowing of food through esophagus, the movement of chyme in entire gastro intestinal tract, the transport of spermatozoa in the cervical canal, transport of bile in bile duct, transport of cilia and is widely applicable in industries where direct contact with boundaries are prohibited. Some examples include finger, hose and roller pumps, food and beverage industry, cell separation, mining, pharamaceutical industry also some bio-mechanical instruments are heart-lung machine, blood pump machine and dialys is machine. Some studies in this direction can be consulted in Refs. [13] [25].

Latham [1] was the first initiated the concept of peristaltic mechanism in 1966, a number of analytic, numerical and experimental studies of peristaltic flow of different fluids have been reported under different conditions with reference to physiological and mechanical situations. After the work of Latham [1], Jaffrin and Shapiro [23] investigated the peristaltic pumping. They made the study under the assumption of long wavelength and low Reynolds number approximation. 
This work further extended for Newtonian and non-Newtonian fluids different flow geometries and boundary conditions as elaborated in the References [2-6][24].

Mixed convection or combined convection is also one of the transport phenomena, is the composition of both natural convection and forced convection flows. Mixed convection flows are encountered in several industrial applications such as nuclear reactors cooled during emergency shutdown, solar central receiver exposed to the wind currents, electronic devices cooled by fans and heat exchangers placed in low velocity environments. Combination of heat and mass transfer in mixed convection flows have special relevance to engineering related problems that include energy from both metal and polymer sheets. Some recent works in the direction can be seen by the investigation [26-30][32].

Nanofluid is a fluid containing nanometer sized particles called nano particles. The nano particles used in nonofluids are typically made of metals, oxides, carbides or carbon nanotubes, nanofibers, droplet, nanosheet etc. peristalsis in connection with nanofluids has application in biomedicines, i.e cancer treatment radio therapy etc. Chio [7] was the first to introduce the word nanofluid that represents the fluid in which nanoscale particles (diameter less than 50nm) are suspended in the base fluid. Recent articles on the nanofluid are cited in Refs[29][31]. Nanofluid has become now the major attention of various researchers for the new manufacturing of automotive and plant cooling systems and for transfer of heat in different heat exchanger devices. Recently a detailed examination of nanofluid was discussed by Akbar et al. [8] the slip effects on the peristaltic transport of nanofluid in an asymmetric channel. The effects of endoscope on the peristaltic transport of nanofluids have been studied by Akbar and Nadeem [9]. Recently, the influence of wall properties on the peristaltic flow of a nanofluid is discussed by Mustafa et al. [10].

Powell and Eyring proposed a new fluid model in 1944 known as Eyring-Powell fluid model [11]. Viscoelastic fluids in physiology and industry have prominent place. The Eyring-Powell nanofluid is one such model which is advantageous to recover accurate results of viscous nanofluid at low and high shear rates. Possibly the complex mathematical description of this model in flexible curved channel averts study of nanoparticles subject to Eyring-Powell fluid [12]. Further investigation of mixed convection peristaltic flow of Eyring-Powell nanofluid in a channel with compliant walls by Anum Tanveer, Hayat $\mathrm{T}$ et al [13]. Nooren S and Qasim [14] studied the peristaltic flow of MHD Eyring-Powell fluid in a channel.

The aim of present work is to study the mixed convection peristaltic flow of Eyring-Powell nanofluid under the effect of magnetic field in a non uniform channel with the consideration of boundary conditions. The equations have been constructed by employing the wave frame and then reduced by assumptions of long wavelength and low Reynolds number approximation. The closed form of the velocity, concentration and temperature solutions are obtained analytically with help of the relationship between them. Finally the physical parameters are discussed in detail via graphs.

\section{Mathematical Formulation}

Let us consider the peristaltic flow of Eyring-Powell nanofluid through a two dimensional non-uniform channel with the sinusoidal wave propagating towards down its walls. The nanofluid is electrically conducting by an external magnetic field $B_{0}$. Here we consider the Cartesian coordinate system $(\tilde{X}, \tilde{Y})$ such that $\tilde{X}$-axis is considered along the centre line in the direction of wave propagation and $\tilde{Y}$-axis is transverse. The geometry of the wall surface can be written as

$$
\tilde{h}(\tilde{X}, \tilde{t})=a(\tilde{X})+b \sin \left(\frac{2 \pi}{\lambda}(\tilde{X}-c \tilde{t})\right)
$$

Here $a(\tilde{X})=a_{20}+k \tilde{X}$ is the channel half width, $\lambda$ is the wavelength, $\tilde{t}$ is the time and $b$ represents the wave amplitude.

The velocity components $\widetilde{U}$ and $\tilde{V}$ along the $\tilde{X}$ and $\tilde{Y}$ directions, respectively, in the fixed frame, the velocity field $V$ is taken as

$$
V=[\tilde{U}(\tilde{X}, \tilde{Y}, \tilde{t}), \tilde{V}(\tilde{X}, \tilde{Y}, \tilde{t}), 0]
$$


The shear of non-Newtonian fluid is considered to the study of Eyring-Powell fluid model. The stress tensor of Eyring-Powell fluid model is [15-17].

$$
\tilde{S}=\mu \nabla \tilde{V}+\frac{1}{\beta} \sinh ^{-1}\left(\frac{1}{c^{*}} \nabla \tilde{V}\right)
$$

Where $\mu$ is viscosity, $\beta$ and $c^{*}$ are the material constant of Eyring-Powell fluid.

Thus, equations embodying the conservation principles of mass, momentum, energy and nanoparticle mass transfer for the governing flow problem of an Eyring-Powell nanofluid can be written as

The continuity equation:

$$
\frac{\partial \tilde{U}}{\partial \tilde{X}}+\frac{\partial \tilde{U}}{\partial \tilde{Y}}=0
$$

The momentum equation:

$$
\begin{aligned}
\rho_{f}\left(\frac{\partial \tilde{U}}{\partial \tilde{t}}+\tilde{U} \frac{\partial \tilde{U}}{\partial \tilde{X}}+\tilde{V} \frac{\partial \tilde{U}}{\partial \tilde{Y}}\right)= & -\frac{\partial \tilde{p}}{\partial \tilde{X}}+\left(\mu+\frac{1}{\beta c^{*}}\right)\left(\frac{\partial^{2} \tilde{U}}{\partial \tilde{X}^{2}}+\frac{\partial^{2} \tilde{U}}{\partial \tilde{Y}^{2}}\right)-\sigma B_{0}{ }^{2} \tilde{U} \\
& -\frac{1}{2 \beta c^{* 3}}\left\{\left(\frac{\partial \tilde{U}}{\partial \tilde{X}}\right)^{2}+\left(\frac{\partial \tilde{U}}{\partial \tilde{Y}}\right)^{2}+2\left(\frac{\partial \tilde{U}}{\partial \tilde{X}}\right)\left(\frac{\partial \tilde{U}}{\partial \tilde{Y}}\right)\right\} \\
& +\rho_{f} g \kappa\left(\tilde{T}-T_{0}\right)+\rho_{f} g \kappa\left(\tilde{C}-C_{0}\right) \\
\rho_{f}\left(\frac{\partial \tilde{V}}{\partial \tilde{t}}+\tilde{U} \frac{\partial \tilde{V}}{\partial \tilde{X}}+\tilde{V} \frac{\partial \tilde{V}}{\partial \tilde{Y}}\right)= & -\frac{\partial \tilde{p}}{\partial \tilde{Y}}+\left(\mu+\frac{1}{\beta c^{*}}\right)\left(\frac{\partial^{2} \tilde{V}}{\partial \tilde{X}^{2}}+\frac{\partial^{2} \tilde{V}}{\partial \tilde{Y}^{2}}\right)-\sigma B_{0}{ }^{2} \tilde{V} \\
& -\frac{1}{2 \beta c^{* 3}}\left\{\left(\frac{\partial \tilde{V}}{\partial \tilde{X}}\right)^{2}+\left(\frac{\partial \tilde{V}}{\partial \tilde{Y}}\right)^{2}+2\left(\frac{\partial \tilde{V}}{\partial \tilde{X}}\right)\left(\frac{\partial \tilde{V}}{\partial \tilde{Y}}\right)\right\}
\end{aligned}
$$

The energy equation

$$
\begin{aligned}
(\rho c)_{f}\left(\frac{\partial \tilde{T}}{\partial \tilde{t}}+\tilde{U} \frac{\partial \tilde{T}}{\partial \tilde{X}}+\tilde{V} \frac{\partial \tilde{T}}{\partial \tilde{Y}}\right)= & k\left(\frac{\partial^{2} \tilde{T}}{\partial \tilde{X}^{2}}+\frac{\partial^{2} \tilde{T}}{\partial \tilde{Y}^{2}}\right)+(\rho c)_{p} D_{B}\left(\frac{\partial^{2} \tilde{C}}{\partial \tilde{X}^{2}}+\frac{\partial^{2} \tilde{C}}{\partial \tilde{Y}^{2}}\right)\left(\frac{\partial^{2} \tilde{T}}{\partial \tilde{X}^{2}}+\frac{\partial^{2} \tilde{T}}{\partial \tilde{Y}^{2}}\right) \\
& +(\rho c)_{p} \frac{D_{T}}{T_{0}}\left(\frac{\partial^{2} \tilde{T}}{\partial \tilde{X}^{2}}+\frac{\partial^{2} \tilde{T}}{\partial \tilde{Y}^{2}}\right)^{2}
\end{aligned}
$$

The concentration equation

$$
\frac{\partial \tilde{C}}{\partial \tilde{t}}+\tilde{U} \frac{\partial \tilde{C}}{\partial \tilde{X}}+\tilde{V} \frac{\partial \tilde{C}}{\partial \tilde{Y}}=D_{B}\left(\frac{\partial^{2} \tilde{C}}{\partial \tilde{X}^{2}}+\frac{\partial^{2} \tilde{C}}{\partial \tilde{Y}^{2}}\right)+\frac{D_{T}}{T_{0}}\left(\frac{\partial^{2} \tilde{T}}{\partial \tilde{X}^{2}}+\frac{\partial^{2} \tilde{T}}{\partial \tilde{Y}^{2}}\right)
$$

Where $\tilde{p}$ is the pressure, $\rho_{f}$ is the density of the fluid, $\sigma$ is the electrical conductivity of the fluid, $B_{0}$ is the applied magnetic field, $g$ is the acceleration due to gravity, $\kappa$ is the volume expansion coefficient, $\widetilde{T}$ is the temperature of the fluid, $\tilde{C}$ is the nanoparticle concentration, $(\rho c)_{f}$ is the heat capacity of the fluid, $k^{*}$ is the thermal conductivity, $(\rho c)_{p}$ is the effective heat capacity of the nanoparticle material, $D_{B}$ is the Brownian diffusion coefficient and $D_{T}$ is Thermophoresis diffusion, $T_{0}$ is the fluid mean temperature.

The relations between the laboratory and wave frame are introduced through

$$
\begin{array}{ll}
\tilde{x}=\tilde{X}-c \tilde{t}, & \tilde{y}=\tilde{Y} \\
\tilde{u}(\tilde{x}, \tilde{y})=\tilde{U}-c, & \tilde{v}(\tilde{x}, \tilde{y})=\tilde{V}
\end{array}
$$

Where $(\tilde{u}, \tilde{v})$ and $(\tilde{x}, \tilde{y})$ indicate the velocity components and coordinates in the wave frame. 
The corresponding boundary conditions for the above problem

$$
\begin{gathered}
\tilde{\psi}=0, \quad \tilde{u}=\frac{\partial \psi}{\partial y}=0, \tilde{T}=0, \tilde{C}=0, \text { at } \tilde{y}=0 \\
\tilde{\psi}=q, \quad \tilde{u}=\frac{\partial \psi}{\partial y}=-c, \tilde{T}=T_{0}, \tilde{C}=C_{0}, \text { at } \tilde{y}=\tilde{h}=a(\tilde{x})+b \sin \frac{2 \pi}{\lambda}(\tilde{x})
\end{gathered}
$$

Now introducing the following non-dimensional quantities

$$
\begin{aligned}
& \psi=\frac{\tilde{\psi}}{c a}, B=\frac{1}{\mu \beta c^{*}}, A=\frac{B c^{2}}{2 a^{2} c^{* 2}}, X=\frac{\tilde{X}}{\lambda}, x=\frac{\tilde{x}}{\lambda}, Y=\frac{\tilde{Y}}{a}, y=\frac{\tilde{y}}{a}, t=\frac{c \tilde{t}}{\lambda}, p=\frac{a^{2} \tilde{p}}{c \lambda \mu}, \\
& v=\frac{\tilde{v}}{c}, \delta=\frac{a}{\lambda}, u=\frac{\tilde{u}}{c}, \operatorname{Re}=\frac{2 \rho_{f} c a}{\mu}, M=\sqrt{\frac{\sigma}{\mu}} B_{0} a, \beta^{*}=\frac{k^{*}}{(\rho c)_{f}}, \operatorname{Pr}=\frac{v}{\beta^{*}}, F=\frac{q}{c a} \\
& G r=\frac{\rho_{f} g k a^{2} \tilde{T}_{0}}{c \mu}, B r=\frac{\rho_{f} g k a^{2} \tilde{C}_{0}}{c \mu}, N b=\frac{(\rho c)_{p} D_{B} \tilde{C}_{0}}{(\rho c)_{f} v}, N t=\frac{(\rho c)_{p} D_{T} \tilde{T}_{0}}{(\rho c)_{f} T_{m} v} \\
& \Omega=\frac{\tilde{C}-\tilde{C}_{0}}{\tilde{C}_{0}}, \theta=\frac{\tilde{T}-\tilde{T}_{0}}{\tilde{T}_{0}}, h=\frac{\tilde{h}}{a_{20}}=1+\frac{\lambda k x}{a_{20}}+\alpha \sin 2 \pi x, \alpha=\frac{b}{a_{20}}
\end{aligned}
$$

The non-dimensional symbols of the above mentioned quantities: $A$ and $B$ are the non-dimensional Eyring-Powell fluid parameters, $\mathrm{Pr}$ is the prandtl number, $\mathrm{Gr}$ and $\mathrm{Br}$ are the Grashof numbers corresponding to the local temperature and local nanoparticles mass transfer respectively, $N b$ is the Brownian motion parameter, $N t$ is the thermophoresis parameter, the Hartmann number $M$, the temperature distribution $\theta, \Omega$ is the mass concentration, $p$ is the dimensionless pressure, $\psi$ is the stream function, $x$ is the non-dimensional axial coordinate, $y$ is the non-dimensional transvers coordinate, $(u, v)$ is the velocity components, $F$ is the dimensionless average flux in the wave frame, $v$ is the nanofluid kinematic viscosity, $\alpha$ is the amplitude ratio.

The nonlinear terms in the momentum equation are determined to be $\operatorname{zero}\left(\operatorname{Re} \delta^{2}\right)$, where $\operatorname{Re}=\frac{2 \rho_{f} c a}{\mu}$ is the Reynolds number and $\delta=\frac{a}{\lambda}$ is the wave number and using non-dimensional quantities and introducing the velocity fields in terms of stream functions $\left(u=\frac{\partial \psi}{\partial y}, v=-\delta \frac{\partial \psi}{\partial x}\right)$ with long wavelength and low Reynolds number approximation, the basic equations (1)-(11) reduces to

$$
\begin{gathered}
\frac{\partial p}{\partial x}=(1+B) \frac{\partial^{3} \psi}{\partial y^{3}}-A\left(\frac{\partial^{2} \psi}{\partial y^{2}}\right)^{3} \frac{\partial^{3} \psi}{\partial y^{3}}-M^{2} \frac{\partial \psi}{\partial y}+G r \theta+B r \Omega \\
\frac{\partial p}{\partial y}=0 \\
\frac{\partial^{2} \theta}{\partial y^{2}}+\operatorname{Pr} N b \frac{\partial \theta}{\partial y} \frac{\partial \Omega}{\partial y}+\operatorname{Pr} N t\left(\frac{\partial \theta}{\partial y}\right)^{2}=0 \\
\frac{\partial^{2} \Omega}{\partial y^{2}}+\frac{N t}{N b} \frac{\partial^{2} \theta}{\partial y^{2}}=0
\end{gathered}
$$

The corresponding dimensionless boundary conditions can be written in the following form 


$$
\begin{aligned}
& \psi=0, \quad \frac{\partial^{2} \psi}{\partial y^{2}}=0, \quad \theta=0, \quad \Omega=0 \quad \text { at } \quad y=0 \\
& \left.\psi=F, \quad \frac{\partial \psi}{\partial y}=-1, \quad \theta=1, \quad \Omega=1 \quad \text { at } \quad y=h=1+\frac{\lambda k x}{a_{20}}+\alpha \sin 2 \pi x\right\}
\end{aligned}
$$

Where the dimensionless time mean flow rate $F$ is the wave frame is related to the dimensionless time mean flow rate $\Theta$ in the laboratory frame as follows

$$
\Theta=F+1, \quad F=\int_{0}^{h} \frac{\partial \psi}{\partial y} d y
$$

Here $\Theta=\frac{Q}{c a}$ and $F=\frac{q}{c a}$ are the dimensionless time mean flow in a fixed and wave form respectively.

Differentiating equation (12) with respect to ' $y$ ', we have

$$
(1+B) \frac{\partial^{4} \psi}{\partial y^{4}}-A \frac{\partial}{\partial y}\left(\left(\frac{\partial^{2} \psi}{\partial y^{2}}\right)^{3} \frac{\partial^{3} \psi}{\partial y^{3}}\right)-M^{2} \frac{\partial^{2} \psi}{\partial y^{2}}+G r \frac{\partial \theta}{\partial y}+B r \frac{\partial \Omega}{\partial y}=0
$$

\section{Solution of the problem}

The solution of the present problem is obtained by using powerful analytical techniques Homotopy Analysis Method (HAM). In the present case we seek initial guess to be [19].

$$
\begin{gathered}
\psi_{0}(y)=\frac{y\left(4 F h^{3}-F y^{3}+h^{4}-h y^{3}\right)}{3 h^{4}} \\
\theta_{0}=\frac{y}{h} \\
\Omega_{0}=\frac{y}{h}
\end{gathered}
$$

The corresponding auxiliary linear operators are

$$
L_{\psi}=\frac{\partial^{5}}{\partial y^{5}}, \quad L_{\theta}=\frac{\partial^{2}}{\partial y^{2}}, \quad L_{\Omega}=\frac{\partial^{2}}{\partial y^{2}}
$$

With property

$$
L_{\psi}\left[C_{1}+C_{2} y+C_{3} \frac{y^{2}}{2}+C_{4} \frac{y^{3}}{6}+C_{5} \frac{y^{4}}{24}\right]=0, \quad L_{\theta}\left[C_{1}+C_{2} y\right]=0, L_{\Omega}\left[C_{1}+C_{2} y\right]=0
$$

Where $C_{1}, C_{2}, C_{3}, C_{4}$ and $C_{5}$ are arbitrary constants, the zeroth-order deformation equations are

$$
\begin{aligned}
& (1-q) L_{\psi}\left[\psi(y ; q)-\psi_{0}(y)\right]=q H_{\psi} h_{\psi} N[\psi(y ; q)] \\
& (1-q) L_{\theta}\left[\theta(y ; q)-\theta_{0}(y)\right]=q H_{\theta} h_{\theta} N[\theta(y ; q)] \\
& (1-q) L_{\Omega}\left[\Omega(y ; q)-\Omega_{0}(y)\right]=q H_{\Omega} h_{\Omega} N[\Omega(y ; q)]
\end{aligned}
$$

Where $q \in[0,1]$ is the embedding parameter, $h_{\psi}, h_{\theta}$ and $h_{\Omega}$ are auxiliary parameters, $L$ is an auxiliary linear operator and $H_{\psi}, H_{\theta}$ and $H_{\Omega}$ are auxiliary functions. $N$ is a non-linear operator and $\psi(y ; q), \theta(y ; q)$ and $\Omega(y ; q)$ are unknown functions and $\psi_{0}(y), \theta_{0}(y)$ and $\Omega_{0}(y)$ are initial approximations of $\psi(y), \theta(y)$ and $\Omega(y)$. It is obvious that when $q=0$ and $q=1$. 
From equation (24) - (26) we can easily found the system of equations with their relevant boundary conditions. According to the methodology of homotopy analysis method the solutions are given by

$$
\begin{array}{r}
\theta(y, q)=\theta_{0}+h_{\theta} \theta_{1}+h_{\theta}{ }^{2} \theta_{2}+\ldots ., \\
\Omega(y, q)=\Omega_{0}+h_{\Omega} \Omega_{1}+h_{\Omega}{ }^{2} \Omega_{2}+\ldots . \\
\psi(y, q)=\psi_{0}+h_{\psi} \psi_{1}+h_{\psi}{ }^{2} \psi_{2}+\ldots .
\end{array}
$$

The solutions for stream function, temperature and concentration are evaluated using equations (27) -(29) and it can be written as

$$
\begin{gathered}
\theta(y, q)=\frac{y}{h}+2 h_{\theta}(N b+N t)\left(\frac{\operatorname{Pr}}{h^{2}}\right) \frac{y^{2}}{2 !}+h_{\theta}{ }^{2}(N b+N t)\left(\frac{\operatorname{Pr}}{h^{2}}\right) \frac{y^{2}}{2 !}+h_{\theta}{ }^{2} h_{\Omega}(N b+N t)^{2}\left(\frac{\operatorname{Pr}^{2}}{h^{3}}\right) \frac{y^{3}}{3 !} \\
+2 h_{\theta}{ }^{3} N t(N b+N t)^{2}\left(\frac{\operatorname{Pr}^{3}}{h^{4}}\right) \frac{y^{4}}{4 !} \\
\Omega(y, q)=\frac{y}{h}+2 h_{\Omega}\left(1+\frac{N t}{N b}\right) \frac{y}{h}+h_{\Omega}{ }^{2}\left(1+\frac{N t}{N b}\right) \frac{y}{h}+h_{\Omega} h_{\theta} N t\left(1+\frac{N t}{N b}\right)\left(\frac{\operatorname{Pr}}{h^{2}}\right) \frac{y^{2}}{2 !}
\end{gathered}
$$

We introduce the stream function $\psi\left(u=\frac{\partial \psi}{\partial y}, v=-\delta \frac{\partial \psi}{\partial x}\right)$ then we get the velocity

$$
\begin{aligned}
u(y, q) & =\frac{1}{3}+\frac{4 F}{3 h}-\left(\frac{8}{h^{3}}+\frac{8 F}{h^{4}}\right) \frac{y^{3}}{3 !}-32 h_{\psi} A\left(\frac{1}{h^{3}}+\frac{F}{h^{4}}\right) \frac{y^{3}}{3 !}+2 h_{\psi} a_{0} \frac{y^{4}}{4 !}+2 h_{\psi} A\left(\frac{5225472}{1591}\right)\left(\frac{1}{h^{3}}+\frac{F}{h^{4}}\right)^{3} \frac{y^{8}}{8 !} \\
& -32 h_{\psi}{ }^{2}\left(\frac{1}{h^{3}}+\frac{F}{h^{4}}\right) \frac{y^{3}}{3 !}+2 A h_{\psi}{ }^{2} a_{0} \frac{y^{4}}{4 !}+2 h_{\psi}{ }^{2} A\left(\frac{5225472}{1591}\right)\left(\frac{1}{h^{3}}+\frac{F}{h^{4}}\right)^{2} \frac{y^{8}}{8 !} \\
& -16 A(1+B) h_{\psi}{ }^{2}\left(\frac{1}{h^{3}}+\frac{F}{h^{4}}\right) \frac{y^{4}}{4 !}+(1+B) h_{\psi}{ }^{2} a_{0} \frac{y^{5}}{5 !}+(1+B) h_{\psi}{ }^{2} A\left(\frac{5225472}{1591}\right)\left(\frac{1}{h^{3}}+\frac{F}{h^{4}}\right)^{2} \frac{y^{9}}{9 !} \\
& +16 M^{2} h_{\psi}{ }^{2} A\left(\frac{1}{h^{3}}+\frac{F}{h^{4}}\right) \frac{y^{6}}{6 !}-M^{2} h_{\psi}{ }^{2} a_{0} \frac{y^{7}}{7 !}-M^{2} h_{\psi}{ }^{2} A\left(\frac{5225472}{1591}\right)\left(\frac{1}{h^{3}}+\frac{F}{h^{4}}\right)^{2} \frac{y^{11}}{11 !} \\
& +h_{\psi} h_{\theta} G r \frac{\operatorname{Pr}}{h^{2}}(N b+N t) \frac{y^{5}}{5 !}+h_{\Omega} h_{\theta} B r \frac{\operatorname{Pr}}{h^{2}}\left(1+\frac{N t}{N b}\right) \frac{y^{5}}{5 !}
\end{aligned}
$$

Where $a_{0}=\left(8(1+B)\left(\frac{1}{h^{3}}+\frac{F}{h^{4}}\right)+8 M^{2}\left(\frac{1}{h^{3}}+\frac{F}{h^{4}}\right)+\frac{G r}{h}+\frac{B r}{h}\right)$

\section{Results and Discussion}

This section prepared to describe the results of our problem graphically using MATHEMATICA program. It consists of three parts; the first part illustrates the impact of some physical parameters on the velocity distribution. However in the second part the evaluation of temperature distribution with different parameters discussed and the third part illustrates the concentration with different parameters.

\subsection{Velocity profile}

Figures 1 to 8 , is plotted to see the behaviour of different parameters on the velocity distribution $u$. Fig. 1 shows that the velocity decreases with an increase in the Eyring-Powell fluid parameter $A$, which appears with the non-linear term of the governing momentum Fig. 2 includes the effect of Eyring-Powell fluid parameter $B$. velocity appears to increase when $B$ is increased. Fig. 3 captures the effects of Hartman number $M$ on the velocity. Here it is observe that velocity is a decreasing function of $M$. Fig. 4 shows that as Grashof number $G r$ increases the velocity profile increases. Fig. 5 includes the effect of local nanoparticle Grashof number $\mathrm{Br}$. Here velocity appears to decreases when $\mathrm{Br}$ is increased. In 
fig. 6 the effect of Brownian motion parameter $N b$ on velocity is plotted. The velocity appears to increases when Brownian motion parameter $\mathrm{Nb}$ increased. The Fig.7 is the effect thermophoresis parameter Nt. The velocity appears to decreases when thermophoresis parameter $N t$ increased. Fig .8 the effect of Prandtl number Pr, velocity appears to increases when Prandtl number Pr increased.

\subsection{Temperature profile}

Figures 9 to 11, is plotted to see the behaviour of different parameters on the temperature distribution $\theta$.Fig. 9 shows that the temperature increases with an increase in the Brownian motion parameter Nt. Fig. 10 captures the effect of thermophoresis parameter $N t$ on the temperature, the temperature decreases when the thermophoresis parameter increases. Fig. 11 includes the effect of Prandtl number $\mathrm{Pr}$ on the temperature, the temperature decreases when the Prandtl number $\mathrm{Pr}$ increased.

\subsection{Concentration profile}

Figures 12 to 14 describe the variation of the concentration profile for several values of the Prandtl number $P r$, thermophoresis parameter $\mathrm{Nb}$ and the Brownian motion parameter $N t$. Fig .12, we observe that the concentration profile increases with an increase in $\mathrm{Nb}$. Fig .13 captures the effect of thermophoresis parameter $N t$ on the concentration profile, the concentration decreases when the thermophoresis parameter increases. Fig .14 includes the effect of prandtl number $\mathrm{Pr}$ on the concentration, the concentration increases when the Prandtl number Pr increases.

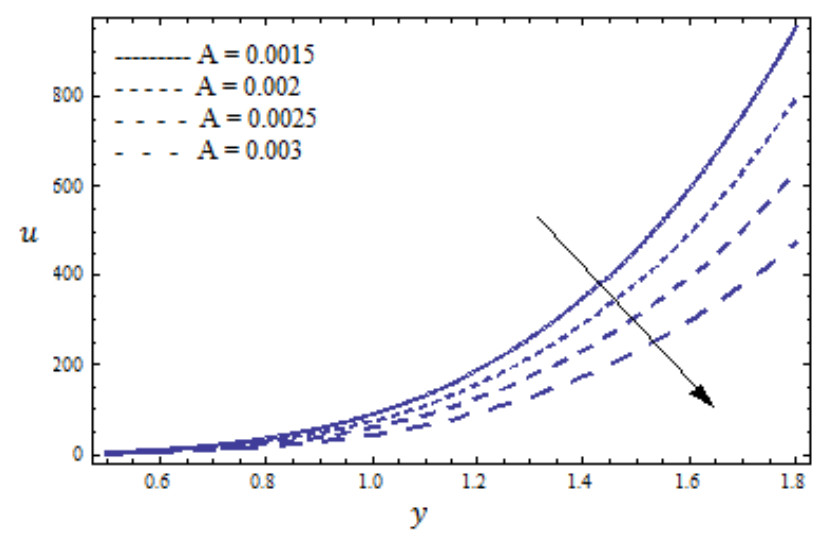

Fig. 1 Velocity profile for different values of $A$ for fixed $B=2.0, M=1.2, G r=0.4, B r=0.3, P r=7.0, N t=0.3, N b=0.2$, $x=0.1, \theta=0.15$.

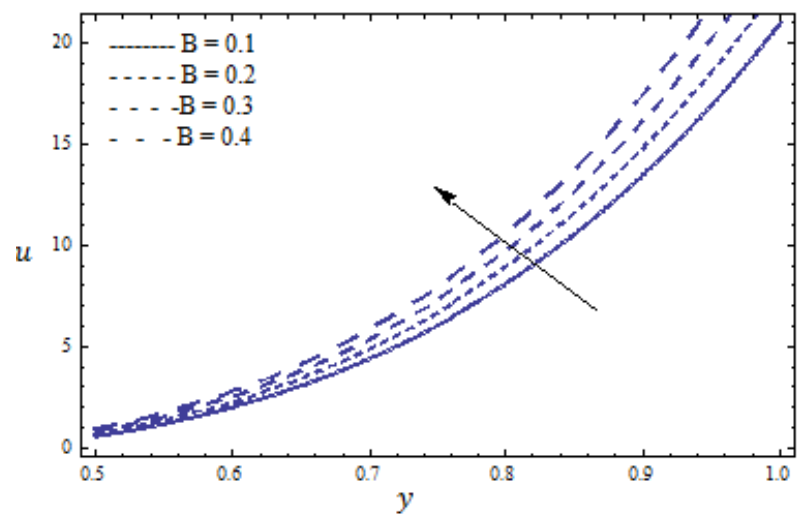

Fig. 2 Velocity profile for different values of $B$ for fixed $A=0.002, M=1.2, G r=0.4, B r=0.3, P r=7.0, N t=0.3, N b=0.2$, $x=0.1, \theta=0.15$. 


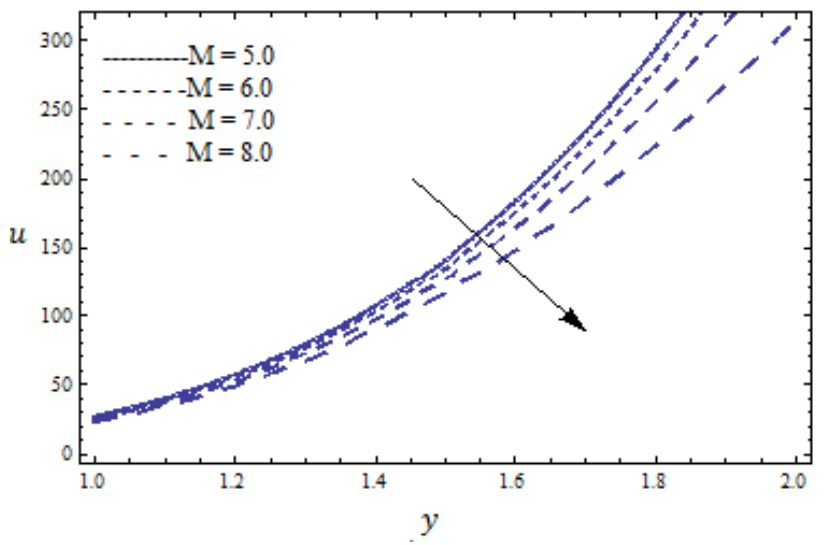

Fig. 3 Velocity profile for different values of $M$ for fixed $A=0.001, B=2.0, G r=0.4, B r=0.3, P r=7.0, N \mathrm{t}=0.3, N b=0.2$, $x=0.1, \theta=0.15$.

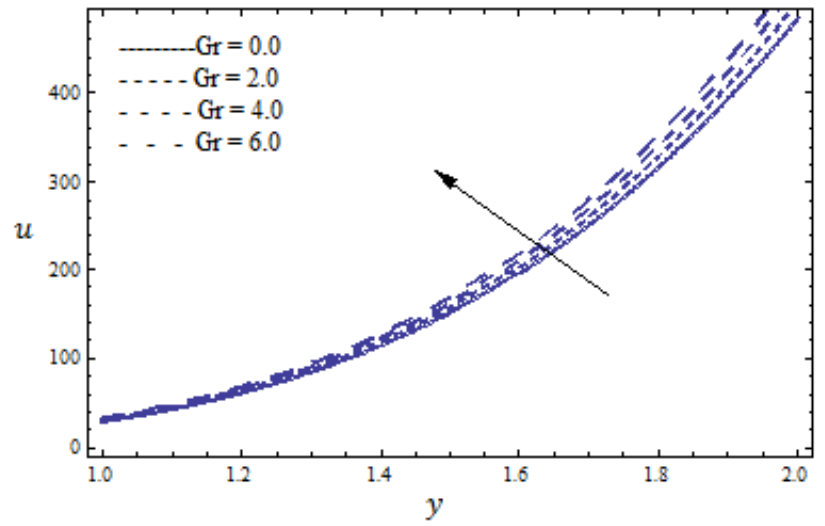

Fig. 4 Velocity profile for different values of $G r$ for fixed $A=0.002, B=0.5, M=0.5, B r=6.0, P r=7.0, N t=0.7, N b=0.6$, $x=0.1, \theta=0.15$.

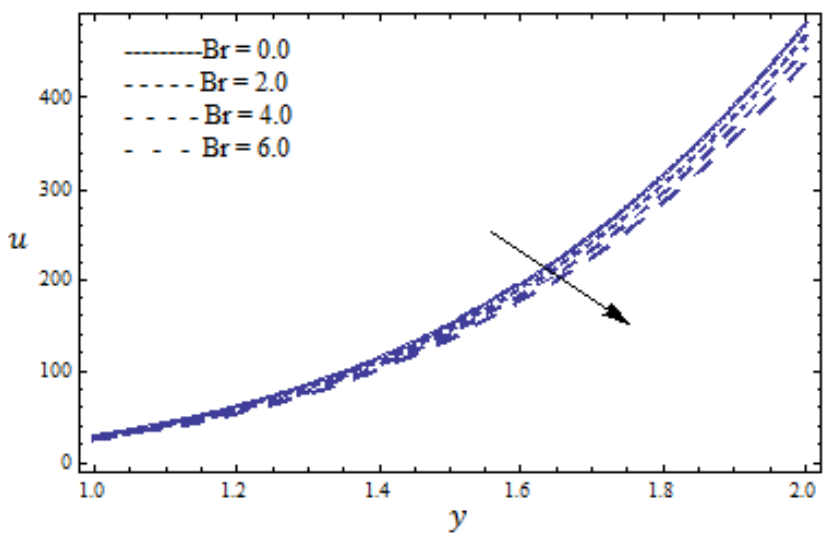

Fig. 5 Velocity profile for different values of $B r$ for fixed $A=0.002, B=0.5, M=0.5, G r=6.0, P r=7.0, N t=0.7, N b=0.6$, $x=0.1, \theta=0.15$. 


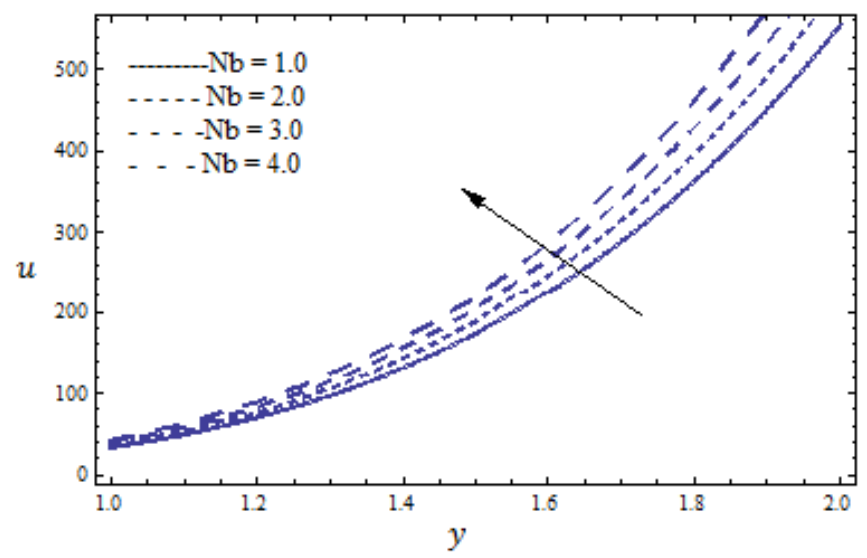

Fig. 6 Velocity profile for different values of $N b$ for fixed $A=0.002, B=0.5, M=0.5, G r=2.0, B r=6.0, P r=7.0$, $N \mathrm{t}=4.0, x=0.1, \theta=0.15$.

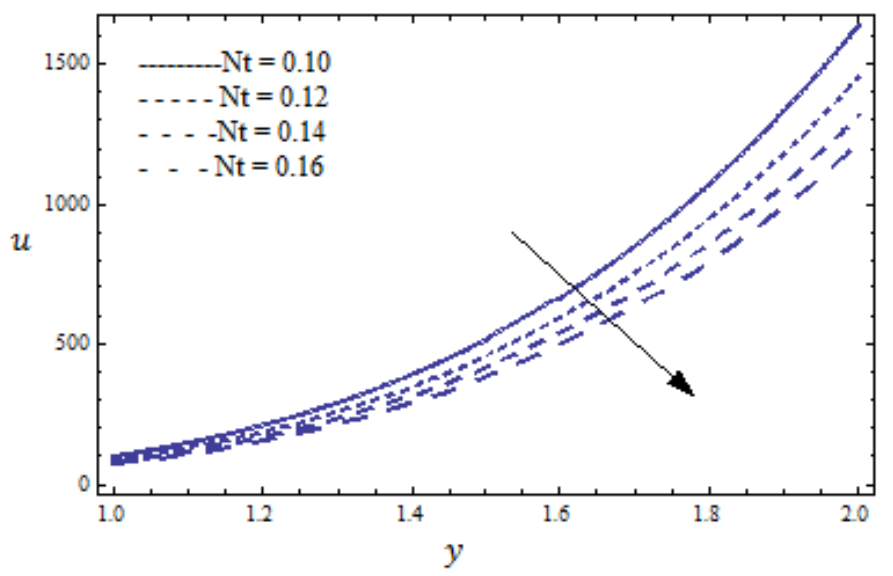

Fig.7 Velocity profile for different values of $N t$ for fixed $A=0.002, B=0.5, M=0.5, G r=2.0, B r=6.0, \operatorname{Pr}=7.0, N b=0.6$, $x=0.1, \theta=0.15$.

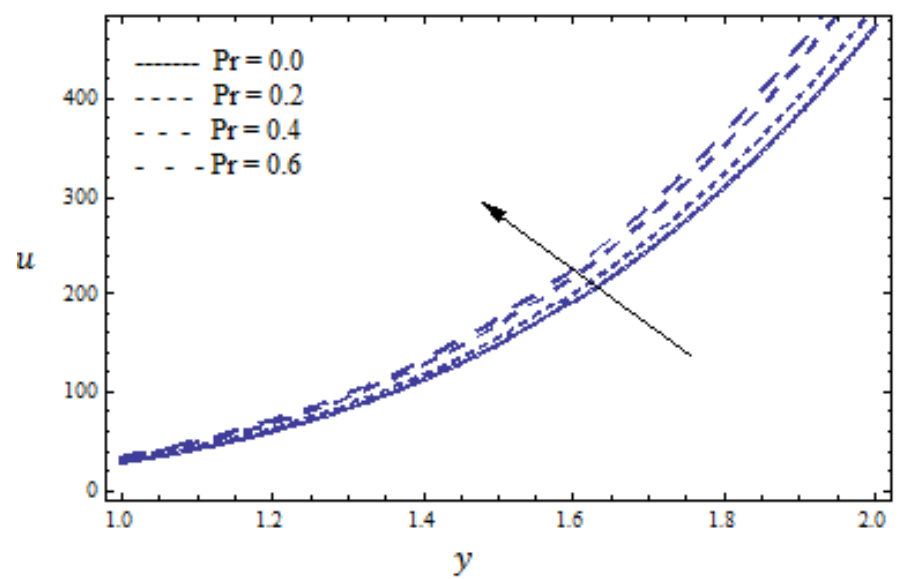

Fig. 8 Velocity profile for different values of $\operatorname{Pr}$ for fixed $A=0.002, B=0.5, M=0.5, G r=2.0, B r=6.0, N b=0.2, N t=4.0$, $x=0.1, \theta=0.15$. 


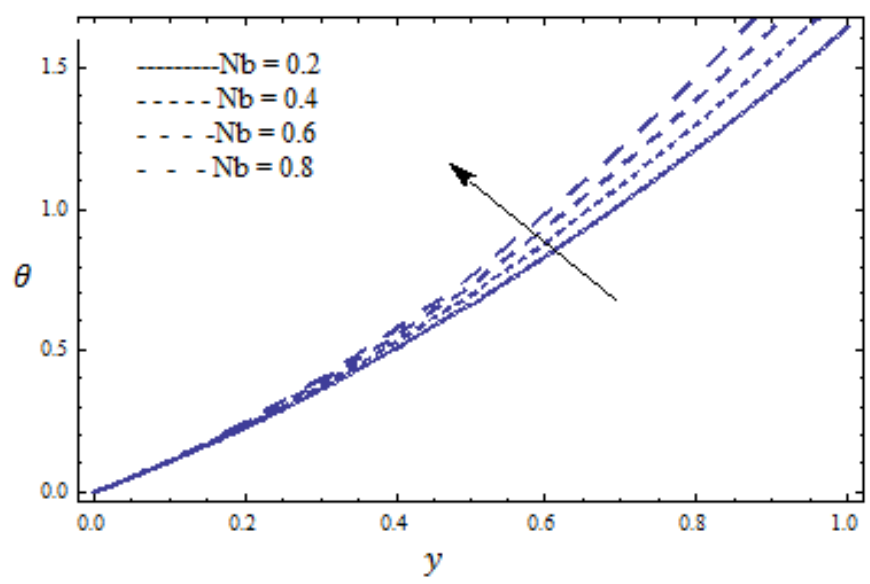

Fig. 9 Temperature profile for different values of $N b$ for fixed $P r=7.0, N t=0.7, x=0.2, \theta=0.15$.

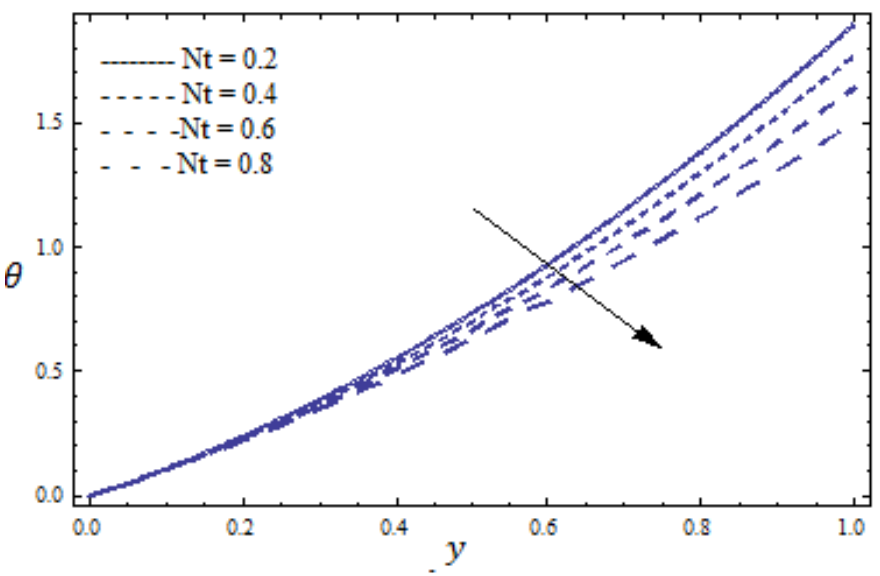

Fig. 10 Temperature profile for different values of $N t$ for fixed $\operatorname{Pr}=7.0, N b=0.5, x=0.2, \theta=0.15$.

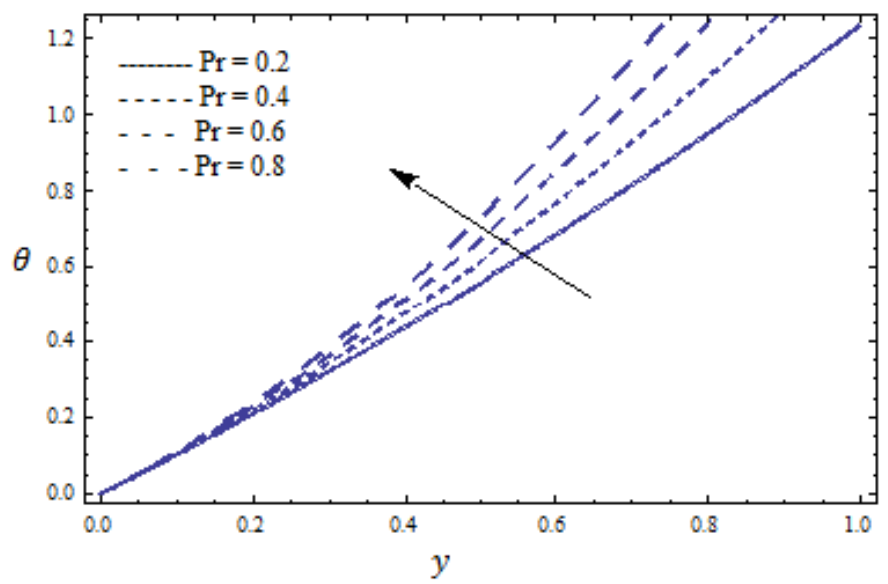

Fig. 11 Temperature profile for different values of $\operatorname{Pr}$ for fixed $N t=0.3, N b=0.5, x=0.2, \theta=0.15$. 


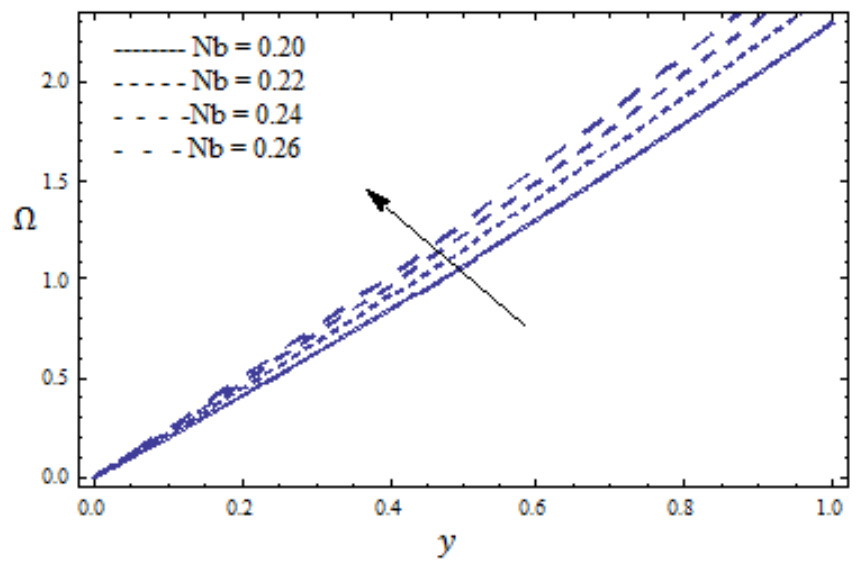

Fig .12 Concentration profile for different values of $N b$ for fixed $N t=0.4, \operatorname{Pr}=7.0, x=0.2, \theta=0.15$.

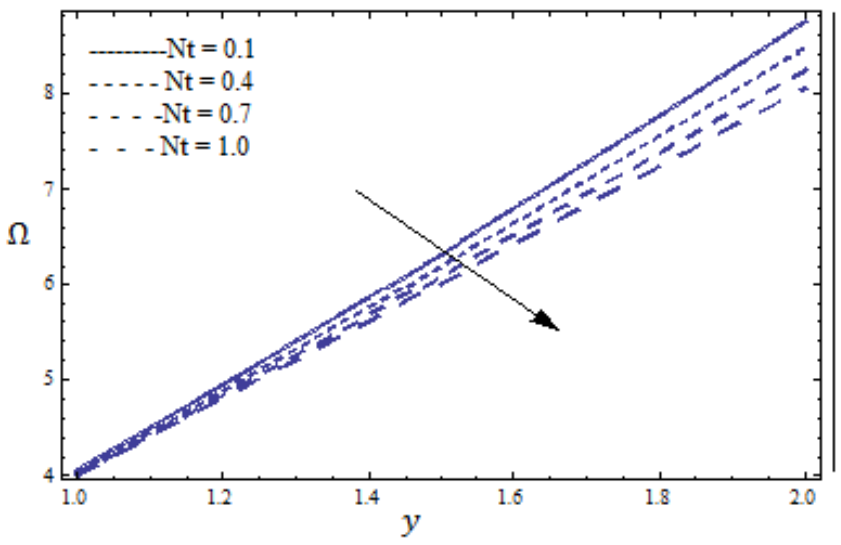

Fig .13 Concentration profile for different values of $N t$ for fixed $N b=4.0, \operatorname{Pr}=7.0, x=0.2, \theta=0.15$.

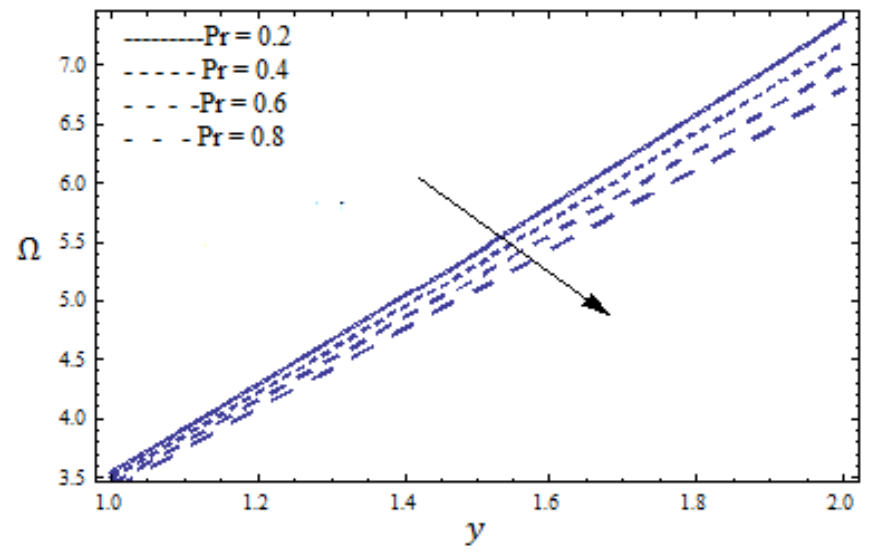

Fig. 14 Concentration profile for different values of $\operatorname{Pr}$ for fixed $N t=0.3, N b=0.5, x=0.2, \theta=0.15$. 


\section{Concluding remarks}

Mixed convection Peristaltic flow of Eyring-Powell nanofluid in a non uniform channel is modelled in the presence of a magnetic field. The main observations of the present analysis are as follows:

- $\quad$ The velocity profile gives opposite results with increasing values of Eyring-Powell fluid parameters $A$ and $B$.

- It is observed that the magnetic parameter $M$ and the Prandtl number $\operatorname{Pr}$ have opposite effects on the velocity.

- Dissimilar response of thermal Grashof number $\mathrm{Gr}$ and local nanoparticle Grashof number $\mathrm{Br}$ are noticed towards velocity of nanofluids.

- Similar behaviour of velocity, temperature and concentration profiles is observed increases with an increase in Brownian motion parameter $\mathrm{Nb}$ and decreases with an increase in the thermophoresis parameter $N t$.

The temperature profile increases with an increase in the Prandtl number $P r$ and the concentration profile decreases with an increase in the Prandtl number $P r$.

\section{References}

[1] Latham T.W.: Fluid motion in a peristaltic pump. MS. Thesis, M.I.T. Cambridge. (1966)

[2] Srinivas S, Gayathri R and Kothandapani M.: The influence of slip conditions, wall properties and heat transfer on MHD peristaltic transport. Comput Phys Commun. 180, 2115-2122 (2009)

[3] Mekheimer K. S.: Peristaltic flow of blood under effect of a magnetic field in a non-uniform channel. Applied Mathematics and Computation. 153, 763-777 (2004)

[4] Asha S K and Rathod V. P.: Effect of magnetic field and an endoscope on peristaltic motion. Advance in applied Science Research. 4, 102-109 (2012)

[5] Asha S K and Rathod V. P.: Peristaltic Transport of magnetic fluid in Uniform and Non-Uniform Annulus. International journal of Mathematics Archive. 12, 1-11 (2012)

[6] Asha S K.: Peristaltic Transport of a magnetic fluid through porous media in a Uniform and Non-Uniform Annulus. Journal of Global Research in Mathematical Archives. 6, 44-54 (2014)

[7] Choi SUS.: Enhancing thermal conductivity of the fluids with nanoparticles. ASME Fluids Eng Div. 231, 99-105 (1995)

[8] Akbar N. S, Nadeem S, Hayat T and Hendi A.A.: Peristaltic flow of a nanofluid with slip effect. Meccania. 47, 1283-1294 (2011)

[9] Akbar N. S and Nadeem S.: Endoscopic effects on peristaltic flow of nanofluid. Commun. Theor. Phys. 56, $761-768$ (2011)

[10] Mustafa M, Hina S, Hayat T and Alsaedi.: Influence of wall properties on the peristaltic flow of a nanofluid: Analytic and Numerical solutions. International Journal of Heat and Mass Transfer. 55, 4871-4877 (2012)

[11]Powell RE and Eyring H.: Mechanism for the relaxation theory of viscosity. Nature. 154, 427-428 (1994)

[12] Abbasi F.M, Alsaedi A and Hayat T.: Peristaltic Transport of Eyring-Powell fluid in a curved channel. J. Aerosp. Eng. (2014)

[13] Anum Tanveer, Hayat T, Fuad Alsaadi and Alsaedi A.: Mixed convection peristaltic flow of Eyring-Powell nanofluid in a curved channel with compliant walls. Computers in Biology and Medicine. 82, 71-79 (2017)

[14]Nooren S and Qasim M.: Peristaltic flow of MHD Eyring-Powell fluid in a channel. Eur. Phys. J. Plus. 91 (2013)

[15] Hayat T, Irfan Shah S, Ahmad B and Mustafa M.: Effect of slip on peristaltic flow of Powell-Eyring fluid in a symmetric channel. Applied Bionics and Biomechanics. 11, 69-79 (2014)

[16] Abbasi F.M, Hayat T and Alsaedi A.: Numerical analysis for peristaltic motion of MHD Eyring-Prandtl fluid in an inclined symmetric cannel with inclined Magnetic field. Journal of Applied Fluid Mechanics.9, 389-396 (2016)

[17] Hina S, Mustafa M, Hayat T and Alsaedi A.: Peristaltic flow of Powell-Eyring in curved channel with heat transfer: A useful application in biomedicine. Computer Methods and Programs in Biomedicine. 135, 89-100 (2016)

[18] Hayat Ali and Ahmed Abdulhadi.: Influence of magnetic field on peristaltic transport for Eyring-Powell Fluid in a Symmetric Channel during a porous medium. Mathematical Theory and Modelling.9, 9-22 (2017)

[19] Mourad S. Semary and Hany N. Hassan.: The Homotopy Analysis Method for Strongly Nonlinear Initial Boundary Value Problems. International Journal of Modern Mathematical Sciences. 3, 154-172 (2014)

[20] Asha S.K. and Sunita G.: Effect of Couple Stress in Peristaltic Transport of Blood Flow by Homotopy Analysis Method. Asian Journal of Science and Technology. 12, 6958-6964 (2017)

[21] Suryanarayana reddy M.: study of MHD effects on peristaltic transport of non-newtonian fluid flows in channels and tubes. JNTUA. (2010)

[22] Komala K.: Peristaltic transport of some non-newtonian fluids through/past porous ducts. VSUT. (2014) 
[23] Jaffrin M. Y and Shapiro A. H.: Peristaltic Pumping .Annual Review of Fluids Mechanics.3, 13-16 (1971)

[24]Nabil T. M Eldabe, Osama M. Abo-Seida et al.: Peristaltic transport of magnetohydrodynamic carreau nanofluid with heat and mass transfer inside asymmetric channel. American Journal of Computational Mathematics 1-20 (2017)

[25] Hayat T, Anum Tanveer et al.: Effects of convective conditions and chemical reaction on peristaltic flow of e yring-powell fluid. 11, 221-233 (2014)

[26] Maria Imtiazi, Hayat T et al.: Mixed convection flow of nanofluid with Newtonian heating. The European physical journal plus. 129, 1-11 (2014)

[27] Sadia Siddiqa and Hossain M.: Mixed convection boundary layer flow over a vertical flat plate with radiative heat transfer. Scientific Research.3, 705-716 (2012)

[28] Rizwan Ul Haq, Zakia Hamouch et al.: MHD mixed convection flow along a vertically heated sheet. International Journal of Hydrogen Energy. 1-8 (2017)

[29] Sadia ayub, Hayat T et al.: Thermal radiation impact in mixed convective peristaltic flow of third grade nanofluid. Results in Physics. 7, 3687-3695 (2017)

[30] Hayat T, Abbasi F.M et al.: MHD Mixed convection peristaltic flow with variable viscosity and thermal conductivity. Sains Malaysiana. 43, 1583-1590 (2014)

[31]Fakour M, Vahabzadeh A et al.: Scrutiny of mixed convection flow of a nanofluid in a vertical channel. Case studies in Thermal Engineering. 4, 15-23 (2014)

[32] Aaiza Gul, Ilyas khan et al.: Heat transfer in MHD mixed convection flow of a ferrofluid along a vertical channel. PLOS ONE. 10, 1-14 (2015) 